\title{
КИНЕМАТИКА ОЧАГА ДЕФОРМАЦИИ ПРИ БЕЗОПРАВОЧНОЙ ПРОКАТКЕ ТРУБ НЕФТЯНОГО СОРТАМЕНТА
}

\author{
А.В. Выдрин ${ }^{1}$, Е.В. Храмков ${ }^{1}$, М.В. Буняшин ${ }^{2}$ \\ ${ }_{1}^{1}$ Южно-Уральский государственный университет, г. Челябинск, \\ ${ }^{2}$ АО «Волжский трубный завод», е. Волжский
}

\begin{abstract}
Рассмотрен процесс безоправочной прокатки труб нефтяного сортамента. Получены зависимости определения параметров калибровки относительно известных обжатия и величины овализации калибра. Обосновано создание математической модели процесса редуцирования и работы извлекательнокалибровочного стана. Приведены зависимости определения основных параметров очага деформации. Получены системы уравнений для нахождения поверхности контакта металла трубной заготовки и инструмента для двухвалкового и трехвалкового калибров. Представлено поле скоростей металла трубной заготовки. Из условия, что осевая компонента вектора скорости изменяется по квадратичному закону и скорость металла на входе очаг деформации со скоростью выхода из очага деформации связаны коэффициентом вытяжки, получена зависимость для определения этой компоненты вектора скорости. Обоснован линейный закон распределения радиальной компоненты вектора скорости металла трубной заготовки, из условия связи осевой компоненты вектора скорости и радиальной тангенсом угла между рассматриваемым сечением и вертикальной плоскостью симметрии валка получена зависимость для определения радиальной составляющей вектора скорости. Третья компонента вектора скорости определена из условия несжимаемости, которое определено для трехвалкового и двухвалкового калибра. На основе определенных компонент вектора скорости определены компоненты тензора скоростей деформации для трехвалкового и двухвалкового калибров. Компоненты тензора скоростей деформации рассчитаны для случая прокатки труб на редукционном трехвалковом стане. Произведена численная оценка компонент тензора скоростей деформации, определены компоненты тензора скоростей деформации, которые возможно не учитывать при проведении инженерных расчетов с использование тензора скоростей деформации.
\end{abstract}

Ключевые слова: кинематика очага деформачии; стан безоправочной прокатки труб; овальная калибровка; тензор скорости деформачии.

В состав большинства современных трубопрокатных агрегатов (ТПА) входят станы для безоправочной прокатки. К таким станам относятся редукционно-растяжные (РРС), извлекательнокалибровочные (ИКС). РРС позволяют увеличить сортамент производимых на ТПА труб за счет дополнительного обжатия по наружному диаметру до $70 \%$, ИКС помимо обжатия по наружному диаметру, также выполняет функцию извлекателя оправки раскатного стана.

Наибольшее распространение получили непрерывные многоклетьевые двух- или трехвалковые станы безоправочной прокатки. Так как они обладают наибольшей технологической гибкостью, достаточно легкой настройкой и имеют простую конструкцию. При этом зачастую используется овальная форма калибров. Такой калибр полностью определяется значениями ширины и высоты калибра, которые в свою очередь определяются значением овализиции и величиной обжатия по наружному диаметру.

При известных ширине $A$ и высоте $B$ калибра

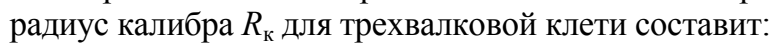

$$
R_{\mathrm{\kappa}}=\frac{A^{2}-A B+B^{2}}{A-2 B} .
$$
(рис. 1):

$$
e=R_{\mathrm{K}}-B \text {. }
$$

Математическое описание процесса прокатки труб на таких станах является важной задачей, поскольку позволит решить ряд задач, таких как определение формоизменения металла в процессе прокатки, энергосиловых параметров и т. п.

В первую очередь при разработке математической модели решаются геометрическая и кинематическая задачи. Известна методика определения геометрических и кинематических параметров очага деформации в декартовой системе координат [1]. Однако ввиду существенной нелинейности заготовки и калибра, при этом получаются достаточно сложные математические зависимости. Гораздо проще вопрос решается в цилиндрической системе координат. Решение геометрической задачи подразумевает символьное определение следующих параметров очага деформации: наружный диаметр трубы $d$ или радиус $r$ в любом сечении очага деформации, длина очага деформации $l$, угол захвата $\alpha_{0}$ (рис. 2).

Общеизвестно, что:

$$
\begin{aligned}
& l=\sqrt{R_{\mathrm{B}}\left(d_{0}-d_{1}\right)} ; \\
& \alpha_{0}=\sqrt{\frac{d_{0}-d_{1}}{R_{\mathrm{B}}}} .
\end{aligned}
$$




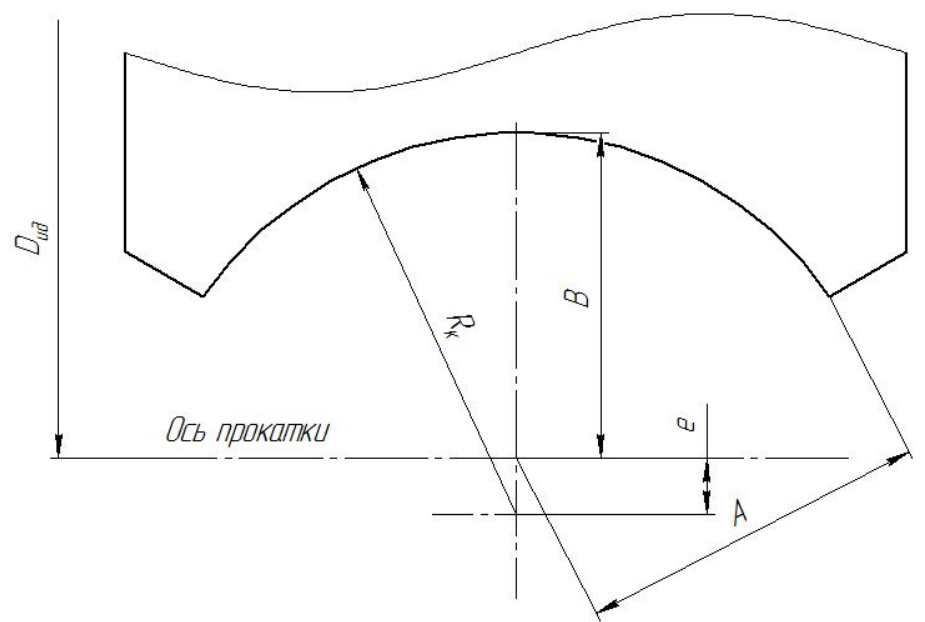

Рис. 1. Схема калибра
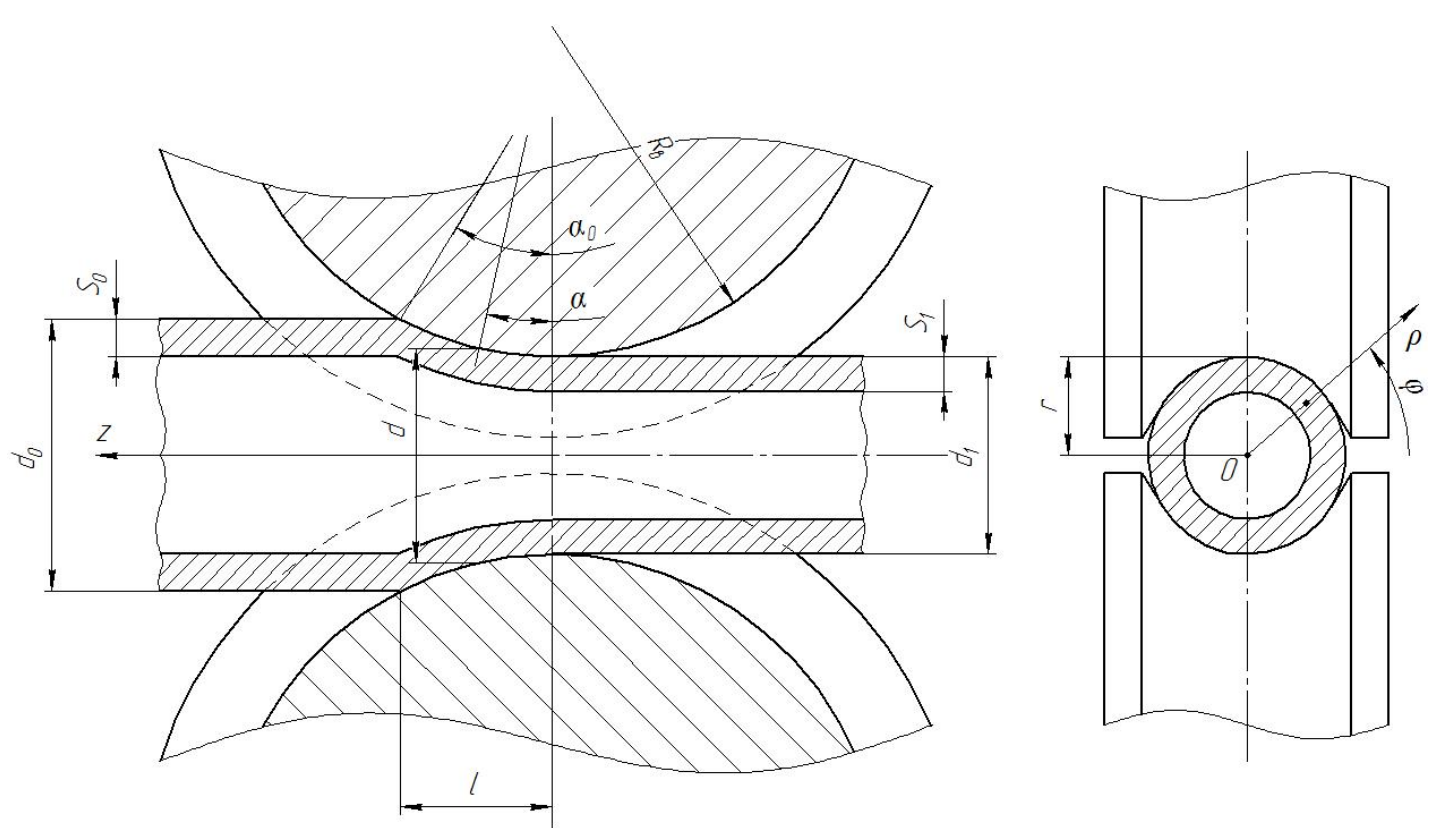

Рис. 2. Схема очага деформации

В соответствие с введенной цилиндрической системой координат наружная поверхность на выходе из очага деформации описывается функцией:

$$
\rho=\sqrt{R_{\mathrm{K}}^{2}-e^{2} \cos ^{2} \varphi}-e \sin \varphi .
$$

В данном случае $\rho$ будет являться наружным радиусом трубы. Поскольку двухвалковый калибр имеет две оси симметрии, а трехвалковый - три, то для двухвалкового можно рассматривать четверть очага деформации $\varphi \in[0 ; \pi / 2]$, для трехвалкового шестую часть: $\varphi \in[\pi / 6 ; \pi / 2]$.

Наружная поверхность трубной заготовки в цилиндрической системе координат описывается функцией:

$$
\rho=r_{0} .
$$

Наружная поверхность валка выражается из уравнения тора:

$$
\begin{aligned}
& \rho \sin \varphi= \\
& =R_{\text {ид }}-\sqrt{\left(R_{\text {ид }}+e-\sqrt{R^{2}-(\rho \cos \varphi)^{2}}\right)^{2}-z^{2}} .
\end{aligned}
$$

Совместное решение уравнений (6) и (7) позволяет определить поверхность контакта металла трубной заготовки с валками первой клети, а совместное решение уравнений (5) и (7) - для последующих клетей. Однако при этом необходимо учитывать, что валки каждой клети развернуты относительно соседних на угол: для двухвалковых клетей $-\pi / 2$, для трехвалковой $-\pi / 3$. То есть при решении системы уравнений (5), (7) в уравнении (5) к ф прибавляется соответствующий угол кантовки.

Решение кинематической задачи при безоправочной прокатке труб нефтяного сортамента (тонкостенных) подразумевает построение поля скоро- 


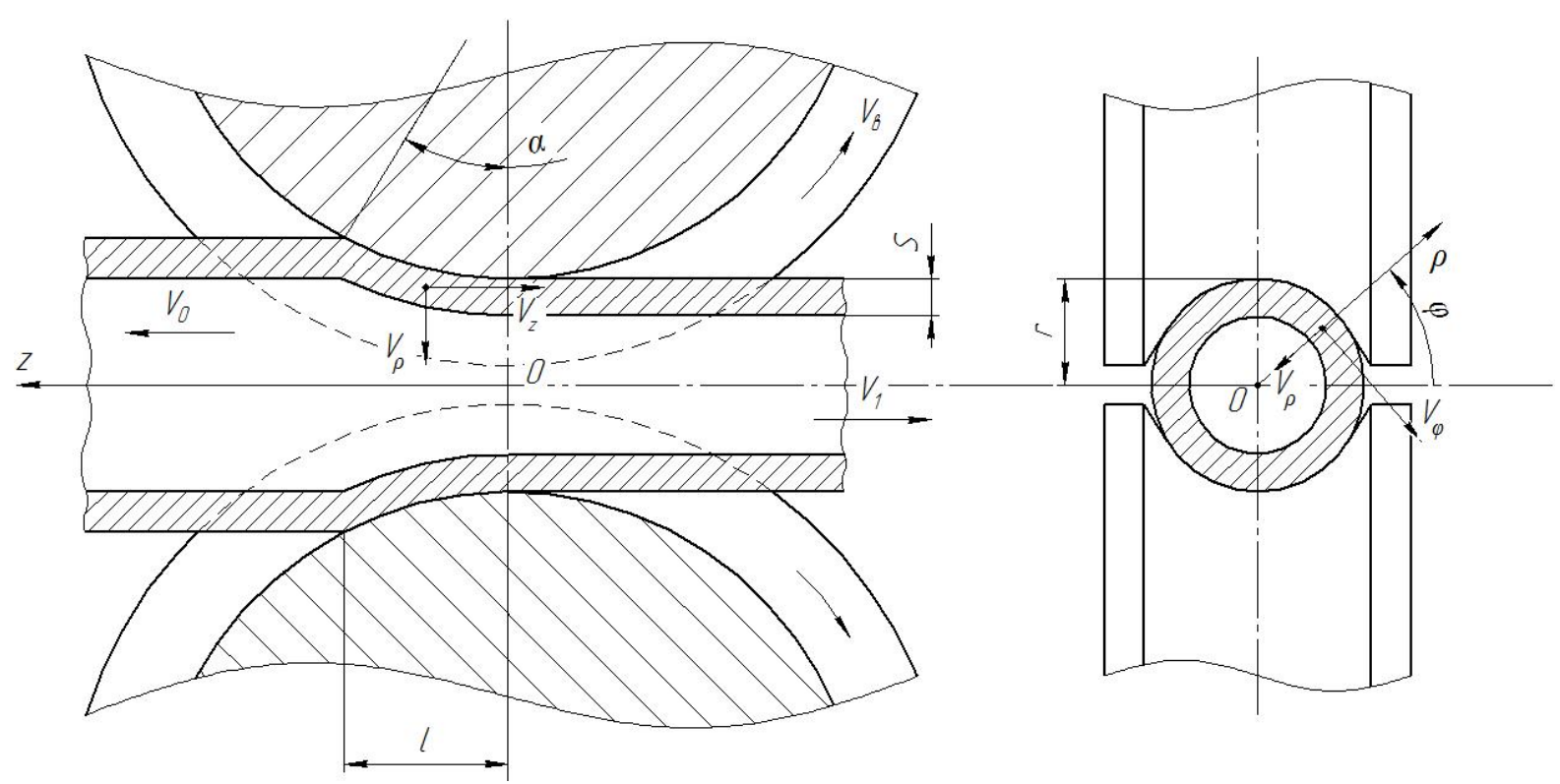

Рис. 3. Компоненты вектора скорости

стей металла трубы и определение тензора скорости деформации.

Вектор скорости в цилиндрической системе координат может быть представлен в виде (рис. 3):

$$
\vec{V}=\left\{\begin{array}{c}
V_{z} \\
V_{\rho} \\
V_{\varphi}
\end{array}\right\} .
$$

Компонента вектора скорости $V_{z}$ в любом поперечном сечении очага деформации должна распределяться равномерно по этому сечению, так как в противном случае появится изгиб или кручение трубы. Поскольку считаем, что прокатный стан настроен правильно, подобные явления должны отсутствовать. Следовательно, данная компонента вектора скорости является функцией координаты $z: V_{z}=V_{z}(z)$.

Поскольку в продольном сечении очага деформации валок имеет форму окружности, принимаем, что компонента вектора скорости $V_{z}$ изменяется вдоль очага деформации по параболическому закону, то есть

$$
V_{z}=a_{0}+a_{1} z+a_{2} z^{2} .
$$

Для определения коэффициентов $a_{0}, a_{1}$ и $a_{2}$ воспользуемся следующим очевидным условием:

$$
\begin{aligned}
& \left.V_{z}\right|_{z=0}=V_{1} ; \\
& \left.V_{z}\right|_{z=l}=V_{0}=\frac{V_{1}}{\lambda} ; \\
& \left.\frac{d V_{z}}{d z}\right|_{z=0}=0,
\end{aligned}
$$

где $V_{1}$ - скорость трубы на выходе из очага деформации; $V_{0}$ - скорость трубы на входе в очаг деформации; $\lambda$ - коэффициент вытяжки.
С учетом этого получим:

$\left.V_{z}\right|_{z=0}=a_{0}=V_{1}$, т. е. $a_{0}=V_{1}$;

$\left.V_{z}\right|_{z=l}=V_{1}+a_{1} l+a_{2} l^{2}=\frac{V_{1}}{\lambda}$, т. e.

$\frac{V_{1}}{\lambda}-V_{1}=a_{1} l+a_{2} l^{2} ;$

из последнего равенства следует:

$$
\begin{aligned}
& \frac{V_{1}}{l}\left(\frac{1-\lambda}{\lambda}\right)=a_{1}+a_{2} l \text { или } a_{1}=\frac{V_{1}}{l}\left(\frac{1-\lambda}{\lambda}\right)-a_{2} l ; \\
& \frac{d V_{z}}{d z}=a_{1}-2 a_{2} z ;\left.\frac{d V_{z}}{d z}\right|_{z=0}=a_{1}=0, \text { т. е. } a_{1}=0,
\end{aligned}
$$

тогда

$$
0=\frac{V_{1}}{l}\left(\frac{1-\lambda}{\lambda}\right)-a_{2} l \text { или } a_{2}=\frac{V_{1}}{l^{2}}\left(\frac{1-\lambda}{\lambda}\right) .
$$

Окончательно для $V_{z}$ можно записать:

$$
\begin{aligned}
& V_{z}=V_{1}+V_{1} \frac{z^{2}}{l^{2}}\left(\frac{1-\lambda}{\lambda}\right) \text { или } \\
& V_{z}=V_{1}\left[1-\frac{z^{2}}{l^{2}}\left(\frac{\lambda-1}{\lambda}\right)\right] .
\end{aligned}
$$

При определении зависимости для $V_{\rho}$ в отличие от поля скоростей, построенного для случая прокатки трубы на оправке [2], будем считать, что $V_{\rho}=0$ при $\rho=0$.

По толщине стенки трубы $V_{\rho}$ теоретически может распределяться по разным зависимостям (рис. 4). Однако ввиду рассмотрения прокатки тонкостенных труб, например, нефтяного сортамента, можно считать, что закон изменения $V_{\rho}(\rho)$ близок к линейному, то есть

$$
V_{\rho}=b_{0}+b_{1} \rho, \text { при } r-s \leq \rho \leq r .
$$




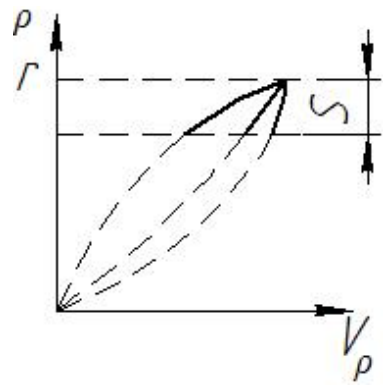

Рис. 4. Распределение компоненты вектора скорости $V_{\rho}$ по толщине стенки

Поскольку труба в поперечном сечении имеет симметричный профиль, компонента вектора скорости $V_{\rho}$ при фиксированной координате $\rho$ будет постоянной по периметру поперечного сечения. Поэтому при определении коэффициентов $b_{0}$ и $b_{1}$ рассмотрим меридиональное сечение трубы, соответствующее вершине калибра ( $\varphi=\pi / 2)$.

Из граничного условия $\left.V_{\rho}\right|_{\rho=0}=0$ следует, что $b_{0}=0$.

На контактной поверхности трубы и валка вектор скорости металла трубы должен быть направлен по касательной к этой поверхности. Это, в свою очередь, определяет соотношение между компонентами вектора скорости $V_{z}$ и $V_{\rho}$ :

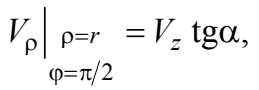

где $\alpha$ - угловая координата произвольной точки на контактной поверхности (см. рис. 1).

Из простых геометрических построений следует, что $\alpha=z / R$, где $R$ - радиус валка по вершине калибра.

Поскольку при деформации труб в станеизвлекателе и редукционных станах обжатия по диаметру относительно малы (как правило, не превышают $10 \%$ ), угол $\alpha$ также достаточно мал, и можно принять:

$$
\operatorname{tg} \alpha \approx \alpha=\frac{z}{R} .
$$

С учетом этого получаем:

$$
\left.V_{\rho}\right|_{\rho=r}=b_{1} r=V_{z} \frac{z}{R} \text { или } b_{1} r=V_{1} \frac{z}{R}\left[1-\frac{z^{2}}{l^{2}}\left(\frac{\lambda-1}{\lambda}\right)\right] \text {. }
$$

Следовательно

$$
b_{1}=\frac{V_{1}}{r} \frac{z}{R}\left[1-\frac{z^{2}}{l^{2}}\left(\frac{\lambda-1}{\lambda}\right)\right] .
$$

Окончательно получим:

$$
V_{\rho}=V_{1} \frac{\rho}{r} \frac{z}{R}\left[1-\frac{z^{2}}{l^{2}}\left(\frac{\lambda-1}{\lambda}\right)\right] .
$$

Компонента вектора скорости $V_{\varphi}$ может быть определена из условия несжимаемости. Условие несжимаемости имеет вид: $\xi=0$, где $\xi$ - скорость относительного изменения объема, которая является первым инвариантом тензора скоростей деформации. Следовательно, можно записать

$$
\xi=\xi_{\rho \rho}+\xi_{\varphi \varphi}+\xi_{z z}=0,
$$

где $\xi_{\rho \rho}, \xi_{\varphi \varphi}, \xi_{z z}$ - компоненты тензора скорости деформации, расположенные на главной диагонали. В цилиндрической системе координат они вычисляются следующим образом [3]:

$$
\begin{aligned}
& \xi_{\rho \rho}=\frac{\partial V_{\rho}}{\partial \rho}=\frac{V_{1}}{r} \frac{z}{R}\left[1-\frac{z^{2}}{l^{2}}\left(\frac{\lambda-1}{\lambda}\right)\right] ; \\
& \xi_{\varphi \varphi}=\frac{\partial V_{\varphi}}{\rho \cdot \partial \varphi}+\frac{V_{\rho}}{\rho}=\frac{\partial V_{\varphi}}{\rho \cdot \partial \varphi}+\frac{V_{1}}{r} \frac{z}{R}\left[1-\frac{z^{2}}{l^{2}}\left(\frac{\lambda-1}{\lambda}\right)\right] ; \\
& \xi_{z z}=\frac{\partial V_{z}}{\partial z}=-V_{1} \frac{2 z}{l^{2}}\left(\frac{\lambda-1}{\lambda}\right) .
\end{aligned}
$$

Отсюда получаем:

$$
\begin{aligned}
& \frac{\partial V_{\varphi}}{\partial \varphi}=\left\{V_{1} \frac{2 z}{l^{2}}\left(\frac{\lambda-1}{\lambda}\right)-\frac{V_{1}}{r} \frac{z}{R}\left[1-\frac{z^{2}}{l^{2}}\left(\frac{\lambda-1}{\lambda}\right)\right]-\right. \\
& \left.-\frac{V_{1}}{r} \frac{z}{R}\left[1-\frac{z^{2}}{l^{2}}\left(\frac{\lambda-1}{\lambda}\right)\right]\right\} \rho
\end{aligned}
$$

или

$$
\begin{aligned}
& V_{\varphi}=V_{1}\left\{\frac{2 z}{l^{2}}\left(\frac{\lambda-1}{\lambda}\right)-\frac{2 z}{r R}\left[1-\frac{z^{2}}{l^{2}}\left(\frac{\lambda-1}{\lambda}\right)\right]\right\} \rho \varphi+C= \\
& =2 \rho \varphi z V_{1}\left\{\frac{1}{l^{2}}\left(\frac{\lambda-1}{\lambda}\right)-\frac{1}{r R}\left[1-\frac{z^{2}}{l^{2}}\left(\frac{\lambda-1}{\lambda}\right)\right]\right\}+C .
\end{aligned}
$$

При прокатке в двухвалковом калибре прокатываемый трубный профиль имеет всегда две оси симметрии, одна из которых лежит в плоскости разъема калибра. Естественно, что благодаря этому тангенциальное течение металла в плоскости разъема калибра будет отсутствовать. Следовательно, для определения константы интегрирования $C$ можно воспользоваться условием $\left.V_{\varphi}\right|_{\varphi=0}=0$, из которого следует, что $C=0$. Таким образом, окончательно для двухвалкового калибра для $V_{\varphi}$ можно записать:

$$
V_{\varphi}=2 \rho \varphi z V_{1}\left\{\frac{1}{l^{2}}\left(\frac{\lambda-1}{\lambda}\right)-\frac{1}{r R}\left[1-\frac{z^{2}}{l^{2}}\left(\frac{\lambda-1}{\lambda}\right)\right]\right\} .
$$

При прокатке в трехвалковом калибре трубный профиль имеет три оси симметрии и для него справедливо следующее: $\left.V_{\varphi}\right|_{\varphi=\pi / 6}=0$, из которого следует, что

$$
C=-2 \rho \frac{\pi}{6} z V_{1}\left\{\frac{1}{l^{2}}\left(\frac{\lambda-1}{\lambda}\right)-\frac{1}{r R}\left[1-\frac{z^{2}}{l^{2}}\left(\frac{\lambda-1}{\lambda}\right)\right]\right\} .
$$

Таким образом, окончательно для трехвалкового калибра для $V_{\varphi}$ можно записать: 


$$
V_{\varphi}=2 \rho\left(\varphi-\frac{\pi}{6}\right) z V_{1}\left\{\frac{1}{l^{2}}\left(\frac{\lambda-1}{\lambda}\right)-\frac{1}{r R}\left[1-\frac{z^{2}}{l^{2}}\left(\frac{\lambda-1}{\lambda}\right)\right]\right\} .
$$

Построенное поле скоростей позволяет определить компоненты тензора скоростей деформации для двухвалкового калибра:

$$
\begin{aligned}
& \xi_{\rho \rho}=\frac{V_{1} z}{r R}\left[1-\frac{z^{2}}{l^{2}}\left(\frac{\lambda-1}{\lambda}\right)\right] ; \\
& \xi_{\varphi \varphi}=2 z V_{1}\left\{\frac{1}{l^{2}}\left(\frac{\lambda-1}{\lambda}\right)-\frac{1}{r R}\left[1-\frac{z^{2}}{l^{2}}\left(\frac{\lambda-1}{\lambda}\right)\right]\right\}+\frac{V_{1} z}{r R}\left[1-\frac{z^{2}}{l^{2}}\left(\frac{\lambda-1}{\lambda}\right)\right] ; \\
& \xi_{z z}=-V_{1} \frac{2 z}{l^{2}}\left(\frac{\lambda-1}{\lambda}\right) ; \\
& \xi_{\rho \varphi}=\frac{1}{2}\left(\frac{\partial V_{\varphi}}{\partial \rho}+\frac{\partial V_{\rho}}{\rho \cdot \partial \varphi}+\frac{V_{\varphi}}{\rho}\right)=\frac{1}{2}\left\{2 \varphi z V_{1}\left(\frac{1}{l^{2}}\left(\frac{\lambda-1}{\lambda}\right)-\frac{1}{r R}\left[1-\frac{z^{2}}{l^{2}}\left(\frac{\lambda-1}{\lambda}\right)\right]\right\rangle+\right. \\
& +0-2 \varphi z V_{1}\left\langle\frac{1}{l^{2}}\left(\frac{\lambda-1}{\lambda}\right)-\frac{1}{r R}\left[1-\frac{z^{2}}{l^{2}}\left(\frac{\lambda-1}{\lambda}\right)\right]\right\rangle=0 ; \\
& \xi_{\varphi z}=\frac{1}{2}\left(\frac{\partial V_{z}}{\rho \cdot \partial \varphi}+\frac{\partial V_{\varphi}}{\partial z}\right)=\frac{1}{2}\left\langle 0+2 \varphi \rho V_{1}\left\{\frac{1}{l^{2}}\left(\frac{\lambda-1}{\lambda}\right)-\frac{1}{r R}\left[1-\frac{z^{2}}{l^{2}}\left(\frac{\lambda-1}{\lambda}\right)\right]\right\}+\right. \\
& \left.+2 \varphi z V_{1} \rho \frac{2 z}{r R l^{2}}\left(\frac{\lambda-1}{\lambda}\right)\right\rangle=\varphi V_{1} \rho\left\{\frac{1}{l^{2}}\left(\frac{\lambda-1}{\lambda}\right)-\frac{1}{r R}\left[1-\frac{z^{2}}{l^{2}}\left(\frac{\lambda-1}{\lambda}\right)\right]+\frac{2 z^{2}}{r R l^{2}}\left(\frac{\lambda-1}{\lambda}\right)\right\} ; \\
& \xi_{z \rho}=\frac{1}{2}\left(\frac{\partial V_{\rho}}{\partial z}+\frac{\partial V_{z}}{\partial \rho}\right)=\frac{1}{2}\left\{V_{1} \frac{\rho}{r R}\left[1-\frac{z^{2}}{l^{2}}\left(\frac{\lambda-1}{\lambda}\right)\right]+V_{1} \frac{\rho z}{r R}\left[-\frac{2 z}{l^{2}}\left(\frac{\lambda-1}{\lambda}\right)\right]+0\right\}=\frac{\rho V_{1}}{2 r R}\left[1-3 \frac{z^{2}}{l^{2}}\left(\frac{\lambda-1}{\lambda}\right)\right] .
\end{aligned}
$$

Для трехвалкового калибра компоненты тензора скоростей деформации не отличаются за исключением $\xi_{\varphi z}$, которая выражается как:

$$
\xi_{\varphi z}=\left(\varphi-\frac{\pi}{6}\right) V_{1} \rho\left\{\frac{1}{l^{2}}\left(\frac{\lambda-1}{\lambda}\right)-\frac{1}{r R}\left[1-\frac{z^{2}}{l^{2}}\left(\frac{\lambda-1}{\lambda}\right)\right]+\frac{2 z^{2}}{r R l^{2}}\left(\frac{\lambda-1}{\lambda}\right)\right\} .
$$

Для численной оценки компонентов тензора скоростей деформации последние были рассчитаны для случая прокатки трубы размерами $73 \times 6$ мм из трубной заготовки $152 \times 6$ мм. На рис. 5 представлена зависимость компонентов тензора скоростей деформации для первой клети от величины обжатия и их распределение по длине очага деформации. При этом настроечные параметры использовались следующие: $\lambda=1,03$, обжатие $-3,2 \% ; V_{0}=1000 \mathrm{Mm} / \mathrm{c}$.

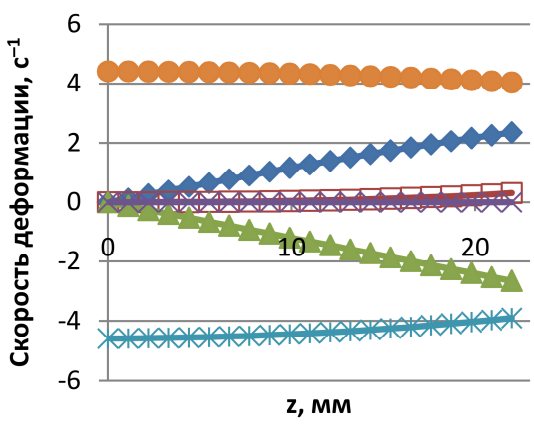

a)

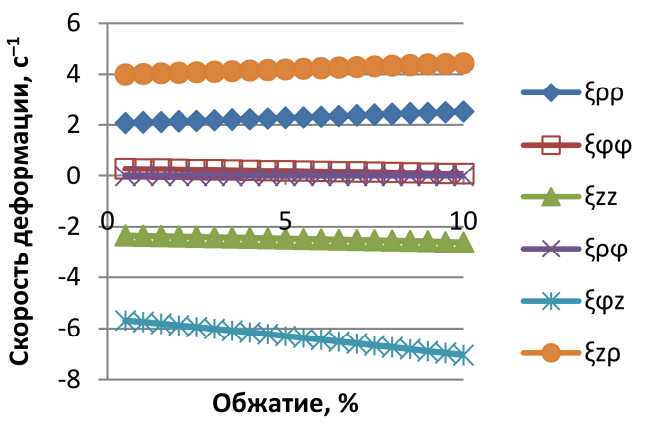

б)

Рис. 5. Компоненты тензора скоростей деформации: а - по длине очага деформации; б - в зависимости от величины обжатия по наружному диаметру

Как видно из рис. 5, б, компонента тензора и $\xi_{\varphi \varphi}$ значительно меньше остальных составляющих, а компонента $\xi_{\rho \varphi}$ равна нулю, что позволяет не учитывать их в инженерных расчетах. Таким образом, определена кинематика очага деформации при безоправочной прокатке труб в двух- и трехвалковом калибрах с точностью до $V_{1}$. 


\section{Лuтература}

1. Блинов, Ю.И. Продольная прокатка труб в многовалковых калибрах / Ю.И. Блинов. - Челябинск: металл, 1992. - 240 с.

2. Выдрин, А.В. Компьютерное моделирование скоростного режима непрерывной прокатки труб / А.В. Выдрин, В.В. Широков // Сталь. - 2011. № 2. - C. 56-58.

3. Колмогоров, В.Л. Механика обработки металлов давлением / В.Л. Колмогоров. - Екатеринбург: Изд-во Уральского государственного технического университета - УПИ., 2001. - 836 с.

Выдрин Александр Владимирович, д-р техн. наук, профессор кафедры машин и технологий обработки материалов давлением, Южно-Уральский государственный университет, г. Челябинск; vydrinav@ susu.ru.

Храмков Евгений Владимирович, аспирант кафедры машин и технологий обработки материалов давлением, Южно-Уральский государственный университет, г. Челябинск; hramkov@rosniti.ru.

Буняшин Михаил Васильевич, начальник центральной заводской лаборатории, АО «Волжский трубный завод», г. Волжский; vtz@vtz.ru.

Поступила в редакцию 6 апреля 2015 2.

DOI: $10.14529 /$ met160113

\title{
KINEMATICS OF THE DEFORMATION ZONE IN PLUGLESS ROLLING OF OIL PIPES
}

\author{
A.V.Vydrin ${ }^{1}$,vydrinav@susu.ru, \\ E.V.Khramkov', hramkov@rosniti.ru, \\ M.V.Bunyashin ${ }^{2}, v t z @ v t z . r u$ \\ ${ }^{1}$ South Ural State University, Chelyabinsk, Russian Federation, \\ 2 JSC "Volzhsky Pipe Plant", Volzhsky, Russian Federation
}

\begin{abstract}
This article describes the process of plugless rolling of oil pipes. Correlations between caliber parameters and swaging were obtained. The mathematical model of the process of plugless rolling of pipes was made. Main parameters of the deformation zone were determined. A set of equations to determine the surface of the contact area of the round billet and the tool for two-roll and three-roll calibers was obtained. The velocity field of metal billets was presented. From the assumption that the axial component of the velocity vector changes according to the quadratic law and the metal entrance velocity in the deformation zone is connected with the exit velocity from the deformation zone by the reduction ratio, the dependence for the determination of this component of the velocity vector was obtained. The linear function of the radial component of the velocity vector was justified. From the relation of axial and radial components of the velocity vector by the tangent of the angle between the considered section and the vertical symmetry plane of the roll a dependence for the determination of the radial component of the velocity vector was defined. The third component of the velocity vector was determined subject to the incompressibility condition, which was defined for three-roll and two-roll calibers. Based on the determined components of the velocity vector, the components of the strain velocity tensor were defined for three-roll and two-roll calibers. The components of the strain velocity tensor were calculated for the case of rolling tubes in a three-roll reduction mill. The components of the strain velocity tensor were numerically estimated, and the components of the strain velocity tensor that may be neglected in engineering calculations were determined.
\end{abstract} tensor.

Keywords: kinematics of deformation zone; plugless tube rolling mill; oval calibration; strain velocity

\section{References}

1. Blinov Yu.I. Prodol'naya prokatka trub v mnogovalkovykh kalibrakh [Longitudinal Rolling of Pipes in Multi-Roll Calibers]. Chelyabinsk, Metall Publ., 1992. 240 p. 
2. Vydrin A.V., Shirokov V.V. Speed Simulation of Continuous Pipe Rolling. Steel in Translation, 2011, vol. 41, no. 2. pp. 140-142. DOI: 10.3103/S0967091211020215

3. Kolmogorov V.L. Mekhanika obrabotki metallov davleniem [Mechanics of Metal Forming]. Ekaterinburg, Ural State Technical University Publ., 2001. 836 p.

Received 6 April 2015

\section{ОБРАЗЕЦ ЦИТИРОВАНИЯ}

Выдрин, А.В. Кинематика очага деформации при безоправочной прокатке труб нефтяного сортамента / А.В. Выдрин, Е.В. Храмков, М.В. Буняшин // Вестник ЮУрГУ. Серия «Металлургия». - 2016. - Т. 16, № 1. - C. 91-97. DOI: 10.14529/met160113

\section{FOR CITATION}

Vydrin A.V., Khramkov E.V., Bunyashin M.V. Kinematics of the Deformation Zone in Plugless Rolling of Oil Pipes . Bulletin of the South Ural State University. Ser. Metallurgy, 2016, vol. 16, no. 1, pp. 91-97. (in Russ.) DOI: $10.14529 / \operatorname{met} 160113$ 